\title{
Inhibitory effects of piperonylic acid on the excessive proliferation of vascular smooth muscle cells and luminal stenosis
}

\author{
Lin L, Hong T \\ The General Hospital of Jinan military Command, Tian Qiao District of Jinan City, Shandong Province. \\ hongtan41@163.com
}

\begin{abstract}
Purpose: Combining in vivo and in vitro experiments, we explored the function and mechanism of piperonylic acid inhibiteing excessive proliferation of vascular smooth muscle cells, and intimal hyperplasia and luminal stenosis after blood vessel injury.

Methods: A model of rat thoracic aorta restenosis after balloon injury was constructed, intragastrically administered with piperonylic acid. 21days later, their thoracic aortas were subjected tests of morphology, SM- $\alpha$-actin, proliferating cell nuclear antigen (PCNA), and expression levels of P21 and P27 by HE staining, immunohistochemistry, and computer image analysis.

Results: The proliferation of vascular smooth muscle cells induced by fetal calf serum was active, and the expressions of P21 and P27 were low. Piperonylic acid obviously promoted the protein expressions of P21 and P27 while inhibiting proliferation and DNA synthesis. After the injury of rat thoracic aorta, the cells moved towards the intima and proliferated excessively, leading to evident neogenesis of intima and luminal stenosis. The SMa-actin immunohistochemistry confirms that the intima contained abundant smooth muscle cells and that the expression of PCNA was high while the expression of P21 and P27 was low. The intervention of piperonylic acid significantly facilitated the gene expressions of P21 and P27, lowered the PCNA expression, and inhibited the formation of intima and the reconstruction of pathological vessels, thus remarkably suppressing luminal stenosis. Conclusion: Piperonylic acid can inhibit the excessive proliferation of vascular smooth muscle cells and the lumen narrowing after injury of blood vessels, the mechanism of which is associated with the promoted gene expressions of cell cycle key regulators P21 and P27 (Tab. 4, Fig. 4, Ref. 22). Text in PDF www.elis.sk.

Key words: piperonylic acid, cell cycle, vascular smooth muscle cell, restenosis, P21, P27, balloon injury.
\end{abstract}

The excessive proliferation of vascular smooth muscle cells (VSMCs) is the main pathological mechanism of vascular proliferative diseases such as atherosclerosis (AS) and restenosis (RS) after percutaneous coronary interventional therapy (PCI). Cells proliferate through the cell cycle which is thus the ultimate common pathway for various stimulating factors to induce the proliferation of VSMCs. P21 and P27, as cyclin-dependent kinase inhibitor (CKIs) exert negative regulation on cell cycle. However, proliferation cell nuclear antigen (PCNA) may be considered as a reliable indicator to reflect the degree of cell proliferation, increased expression of which indicates stronger proliferation capacity, and vice versa (1-4). It is still the focus of current medical research and development to search for a more efficient and low-toxic medicine that can prevent and cure such vascular proliferative diseases.

At present, pure natural medicine has aroused particular attention owing to the unique curative effect, low prize and low toxicity. Piper nigrum contains $60-70 \%$ of piperonylic acid that

The General Hospital of Jinan military Command, Tian Qiao District of Jinan City, Shandong Province

Address for correspondenece: Hong Tan, The General Hospital of Jinan military Command, Normal Road No. 25 Tian Qiao District of Jinan City, Shandong Province is therapeutically valuable. Separated and purified piperonylic acid is water-soluble, secure and nontoxic. Recent researches have verified that piperonylic acid not only inhibited the growth of cancer cells and regulated the immunity, but also improved the lipid metabolism, enhanced endothelial function, and resisted atherosclerotic proliferation. However, related experimental studies are still limited (5-7).

Based on combined in vivo and in vitro experiments, this study observed the effects of piperonylic acid on rat VSMCs proliferation, intimal hyperplasia after aortic balloon injury, vascular remodeling, and luminal stenosis. We also tested the regulation effects of piperonylic acid on the VSMCs DNA synthesis as well as the expressions of key regulators in cell cycle (PCNA, P21, and P27), aiming to explore the influences of piperonylic acid on inhibiting the VSMCs excessive proliferation and the luminal stenosis after injury of blood vessels.

\section{Materials and methods}

\section{Materials}

The main materials and apparatus in this study included: piperonylic acid, Beijing Donghua Rio Tinto Technology Development Co., Ltd.; male SD rats, Shanghai Slac Laboratory Animals Co., 


\section{1-765}

Ltd.; DMEM culture medium, fetal bovine serum (FBS), trypsin, tritiated thymidine ( $\left.{ }^{3} \mathrm{H}-\mathrm{TdR}\right), \mathrm{SP}$ and SLABC kit, Beijing Wanyu Meilan Technology Development Co., Ltd.; DAB and SM- $\alpha$-actin monoclonal antibody, PCNA mouse monoclonal antibody, P21 monoclonal antibody and P27 rabbit polyclonal antibody, Shanghai Youlong Biotech Co,. Ltd. UV spectrometer and 3K30 high-speed low-temperature refrigerated centrifuge, Shanghai AoXi Technology Instrument Co., Ltd.; $311 \mathrm{CO}_{2}$ incubator, Shanghai CIMO Medical Instrument Co., Ltd.; $2 \mathrm{~F}$ balloon catheter, OLYMPUS optical microscope and automatic camera system, and VIDAS computer image analysis system.

\section{Cell experiments}

Culture of VSMCs

Under aseptic conditions, the thoracic aortas of rats (Wistar rats, 180-200 g, male, about 8 weeks old) were disconnected. The medial smooth muscle tissue blocks were transferred into a culture bottle according to the tissue piece method, into which DMEM culture medium was added containing $5 \%$ FBS and cultured in at $37^{\circ} \mathrm{C}$ in $5 \% \mathrm{CO}_{2}$ incubator, and the growth of tissue block edge cell was observed under an inverted microscope. The cells were passaged and cultured after trypsin digestion. Under the light microscope, the cells presented typical "peak-valley", which were then identified by the actin antibody immunohistochemical method as VSMCs. The experiments used 5-7 generations of cells.

\section{Experimental grouping}

Control group: VSMCs in the stationary phase; FBS group: 5 $\%$ FBS was added; the intervention group of piperonylic acid (piperonylic acid+FBS) was divided into 4 subgroups: $5 \%$ FBS and piperonylic acid (20,40,60, $80 \mathrm{mg} / \mathrm{L})$ were added simultaneously. Six parallel sampling wells were set for each group.

\section{Determination of ${ }^{3} \mathrm{H}$-TdR incorporation efficiency}

VSMCs were inoculated on 96-well culture plates $\left(1 \times 10^{6} / \mathrm{L}\right)$, and the culture medium was refreshed after $48 \mathrm{~h}$ and then discarded when $90 \%$ of the cells fused, with serum-free DMEM culture medium used instead. The cells were then starved for $48 \mathrm{~h}$ to be synchronized in the G0 phase. $50 \mu$ of $\mathrm{pH} 7.4$ PBS was added into each well of the control group, $5 \%$ FBS was added into the FBS group, and piperonylic acid solutions (final concentrations: 20, $40,60,80 \mathrm{mg} / \mathrm{L}$ ) were added into each well of each experimental group respectively. $48 \mathrm{~h}$ later, ${ }^{3} \mathrm{H}-\mathrm{TdR}\left(3.0 \times 10^{5} \mathrm{~Bq} / \mathrm{L}\right)$ was added, $48 \mathrm{~h}$ after which the cells were collected. Then the cell membrane was broken, and $3 \mathrm{ml}$ of scintillating solution were added. The radiation intensity (7) was measured by liquid scintillation counting.

\section{Western blot}

The proteins of cells in each group were extracted and quantified. After being separated by $10 \%$ SDS-PAGE, the products were electrically transferred to a nitrocellulose film and blocked by 5 $\%$ bovine serum albumin. After washed, anti-rabbit P21 and P27 polyclonal antibodies (working concentration, 1:50) were added to be cultured for $2 \mathrm{~h}$. After another washing, goat anti-rabbit IgG labeled by horseradish peroxidase (HRP) (working concentration,
1:100) was added to be culture for $2 \mathrm{~h}$. Then DAB was used for development within $30 \mathrm{~min}$. Optical density (A) was determined on a gel image analysis system (VIDAS21).

\section{Animal experiments}

Animal grouping and treatment

24 male SD rats weighing 300-350 g were selected. The rats were randomly divided into 3 groups: (1) Piperonylic acid treatment group: The rats were subjected to balloon-injured thoracic aorta surgeries by being intragastrically administered with $3 \mathrm{ml}$ of piperonylic acid $(10 \mathrm{~g} / \mathrm{L})$ daily since the preoperative day 3 . (2) Surgery control group: The rats were subjected to ballooninjured thoracic aorta surgeries by being intragastrically administered with normal saline. (3) Normal control group: The rats were subjected to sham-operations without inserting balloon catheters while maintaining other surgical conditions unchanged and by being intragastrically administered with normal saline. The rats were executed 21 days later.

Modeling of rat balloon-injured thoracic aorta and preparation of samples

The piperonylic acid treatment group and the surgery control group were anesthetized with pentobarbital sodium $(20 \mathrm{mg} / \mathrm{kg}$, ip), and 2F balloon catheter was inserted through the left common carotid artery. The part from the renal artery bifurcation to the opening of aortic arch and carotid artery was subjected to balloon injury to establish the animal model with exfoliated artery endothelium and injured tube wall. 21days after the operation, the chest was opened to expose the aorta, from which vascular segments $2-3 \mathrm{~cm}$ beyond the diaphragm were taken and put in $5 \%$ neutral buffered formaldehyde solution, fixed for $12 \mathrm{~h}$, and embedded in paraffin (8).

\section{Morphological analysis}

The paraffin sections were stained by HE to measure the below values in the artery cross section by the optical image analyzer: luminal area (LA), intima area (IA), the media area (MA), intima/media area ratio (IA/MA), and internal or external elastic lamina area (IEL, EEL).

\section{Immunohistochemical staining}

Immunohistochemical staining kit was used. First antibody was the rabbit anti-human SM- $\alpha$-actin/PCNA/P21/P27 protein monoclonal antibody. Cytoplasts (SM- $\alpha$-actin) or cell nuclei (PCNA, P21, P27) stained brown (brown-yellow, brown, sepia) were positive. We used image analyzer to randomly measure the average optical density and the percentage of positive cells in 6 visual fields of each section, which were averaged, respectively. Their product multiplied by 100 was utilized as the positive expression index to indicate the expression level of protein. First antibody was replaced with PBS as the negative control.

\section{Statistical analysis}

All data were analyzed by $t$ test and analysis of variance via SPSS 13.0 and expressed as $\mathrm{x} \pm \mathrm{s}$. 


\section{Results}

Effects of piperonylic acid on the synthesis of VSMCs DNA

The experimental results (Tab. 1) of ${ }^{3} \mathrm{H}-\mathrm{TdR}$ incorporation efficiency show that $5 \%$ FBS obviously increased the ${ }^{3} \mathrm{H}-\mathrm{TdR}$ incorporation of VSMCs while piperonylic acid (20, 40, 60, 80 $\mathrm{mg} / \mathrm{L}$ ) apparently decreased the ${ }^{3} \mathrm{H}-\mathrm{TdR}$ incorporation of VSMCs. In addition, the inhibitory effects enhanced with rising concentration. The results suggest that piperonylic acid inhibited the DNA synthesis and proliferation of VSMCs.

Effects of piperonylic acid on the expressions of P21 and P27 (protein grayscale, OPTDI)

The Western blot and image analysis results show (Tab. 2 and Fig. 1) that the VSMCs of the control group barely proliferated, out of which P21 was lowly expressed and P27 was highly expressed. FBS augmented the proliferation of VSMCs, with increased P21 expression and obviously decreased P27 expression. After adding piperonylic acid solutions $(20,40,60,80 \mathrm{mg} / \mathrm{L})$, the protein expressions of P21 and P27 remarkably increased, indicating that piperonylic acid increased the expression of cell cycle inhibitors P21 and P27 to significantly suppress the excessive proliferation of VSMCs.

\section{Vascular morphologies after balloon injury}

The normal control group had smooth interior walls and complete endothelium while the inner membrane comprised single-layer endothelial cells and few extracellular matrix. 21days after the operation, the surgery control group experienced obvious intima formation and lumen stenosis. The SM- $\alpha$-actin immunohistochemical results verify that the new intima was rich in VSMCs (Fig. 2A). Compared with the surgery control group (Fig. 2B), the IA of the piperonylic acid treatment group (Fig. 2C) was reduced by $70.39 \%$, IA/MA was evidently decreased, IEL and EEL were significantly augmented, and LA was elevated by $23.29 \%$ (p < 0.01) (Tab. 3).

Tab. 1. Effects of piperonylic acid on the synthesis of VSMCs DNA.

\begin{tabular}{lc}
\hline Group & 3H-TdR (dpm/well) \\
\hline Control & $1351.35 \pm 365.15$ \\
FBS & $9518.36 \pm 491.58^{*}$ \\
FBS $+20 \mathrm{mg} / \mathrm{L}$ piperonylic acid & $7565.30 \pm 430.26^{*} \#$ \\
FBS $+40 \mathrm{mg} / \mathrm{L}$ piperonylic acid & $6215.22 \pm 505.61^{*} \#$ \\
FBS $+60 \mathrm{mg} / \mathrm{L}$ piperonylic acid & $4913.29 \pm 391.54^{*} \#$ \\
FBS $+80 \mathrm{mg} / \mathrm{L}$ piperonylic acid & $3536.85 \pm 428.27 * \#$ \\
\hline
\end{tabular}

$* \mathrm{p}<0.01$ vs the control group; \# $\mathrm{p}<0.01$, compared with FBS group + piperonylic acid

Tab. 2. Effects of piperonylic acid on the expressions of P21 and P27.

\begin{tabular}{lcc}
\hline Group & P21 (OPTDI) & P27 (OPTDI) \\
\hline Control & $11.25 \pm 3.64$ & $67.55 \pm 8.19$ \\
FBS & $35.61 \pm 9.60^{*}$ & $29.58 \pm 9.15^{*}$ \\
FBS+20 mg/L piperonylic acid & $49.51 \pm 8.36^{* \#}$ & $43.35 \pm 8.54 * \#$ \\
FBS+40 mg/L piperonylic acid & $62.36 \pm 9.42 * \#$ & $57.25 \pm 9.18^{*} \#$ \\
FBS+60 mg/L piperonylic acid & $83.42 \pm 9.45^{* \#}$ & $69.48 \pm 9.38 \#$ \\
FBS+80 mg/L piperonylic acid & $102.25 \pm 10.67 * \#$ & $91.52 \pm 8.17 * \#$ \\
\hline p $<0.01 \mathrm{vs}$ the control group; \# $<0.01$ compared with FBS group + pipero- \\
nylic acid
\end{tabular}

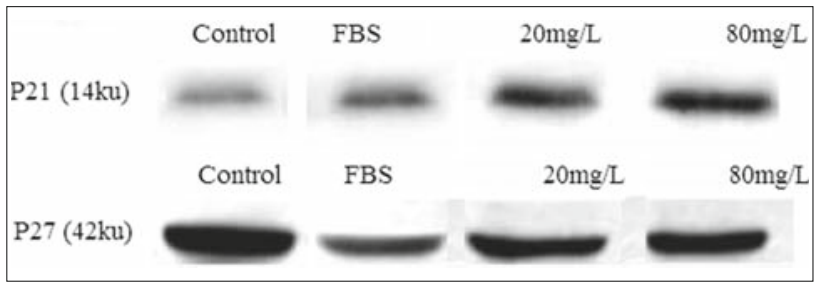

Fig. 1. Effects of piperonylic acid on the expressions of P21 and P27.

Effects of piperonylic acid on PCNA expression after balloon injury

The vessel wall of the normal control group did not express PCNA. 21days after the operation, considerable intima was expressed in the surgery control group. The intimal positive indices of the piperonylic acid treatment group were significantly lower than those of the surgery control group $(\mathrm{p}<0.01)$ (Tab. 4).

\section{Changes of P21 expression after balloon injury}

$\mathrm{P} 21$, which was not expressed in the normal control group, was slightly expressed in the surgery control group 21 days after the operation. A few brown-yellow or brown positive VSMCs were found in the evidently thickened endometrial cavity surface. Contrarily, piperonylic acid treatment dramatically promoted the P21 expression, which was 2.06 times higher than that of the surgery group ( $<<0.01)$ (Tab. 4 and Fig. 3).

Effects of piperonylic acid on P27 expression after balloon injury

P27 was apparently expressed in the normal control group, manifested as brown positively expressed nuclei. 21 days after the operation, only a few brown-yellow or brown positive VSMCs were discerned in the evidently thickened endometrial cavity surface, indicating significantly reduced expression. The P27 positive index in the neointima of piperonylic acid treatment group was 2.03 times higher than that of the surgery group $(\mathrm{p}<$ 0.01 ), suggesting a significantly enhanced P27 expression (Tab. 4 and Fig. 4).

Tab. 3. Effects of piperonylic acid on the formation of new intima and the reconstruction of lumen after balloon injury.

\begin{tabular}{lcc}
\hline Feature $\left(\mathrm{mm}^{2}\right)$ & Surgery control & Piperonylic acid \\
\hline LA & $1.324 \pm 0.148$ & $1.726 \pm 0.151^{*}$ \\
IA & $0.358 \pm 0.054$ & $0.106 \pm 0.018^{*}$ \\
Medium area & $0.451 \pm 0.061$ & $0.436 \pm 0.025$ \\
IA/MA & $0.794 \pm 0.086$ & $0.243 \pm 0.072^{*}$ \\
Internal smooth muscle area & $1.521 \pm 0.084$ & $1.823 \pm 0.103^{*}$ \\
External smooth muscle area & $1.735 \pm 0.125$ & $2.015 \pm 0.116^{*}$ \\
\hline
\end{tabular}

$* \mathrm{p}<0.01$ vs the surgery control group

Tab. 4. Effects of piperonylic acid on the expressions of PCNA, P21 and $P 27$ after balloon injury.

\begin{tabular}{lccc}
\hline Group & PCNA & P21 & P27 \\
\hline Normal control & No expression & No expression & $17.28 \pm 2.66$ \\
Surgery & $18.24 \pm 3.54$ & $7.55 \pm 1.36$ & $8.95 \pm 1.36$ \\
Piperonylic acid & $5.29 \pm 1.25^{*}$ & $15.58 \pm 2.64^{*}$ & $18.15 \pm 2.81^{*}$ \\
\hline
\end{tabular}

$* \mathrm{p}<0.01$ vs the surgery control group 

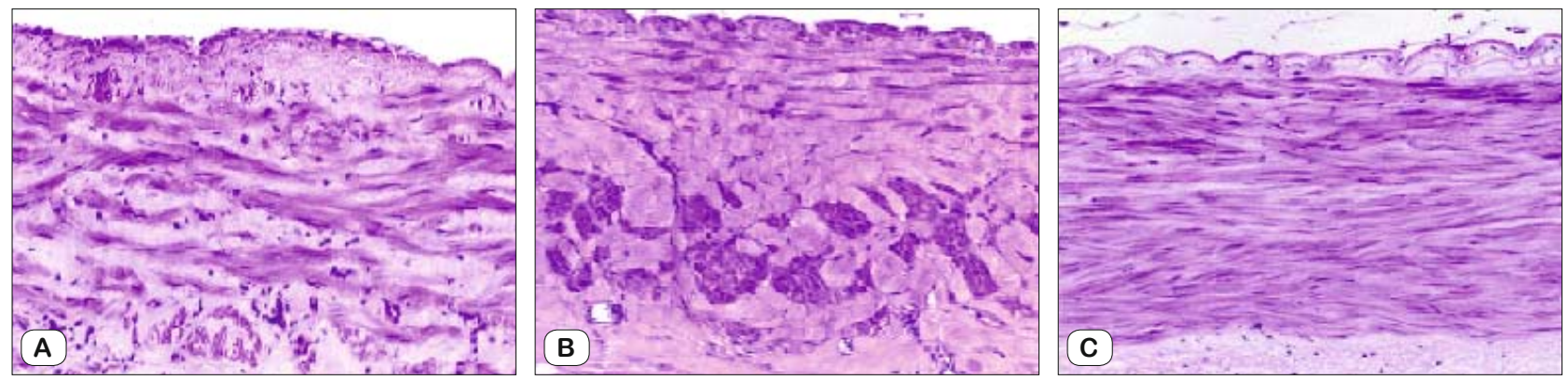

Fig. 2. Vascular morphologies after balloon injury. A: immunohistochemical staining of SM- $\alpha$-actin; B: intima of the surgery group; C: intima of the piperonylic acid treatment group ( $p<0.01$ vs the surgery group).
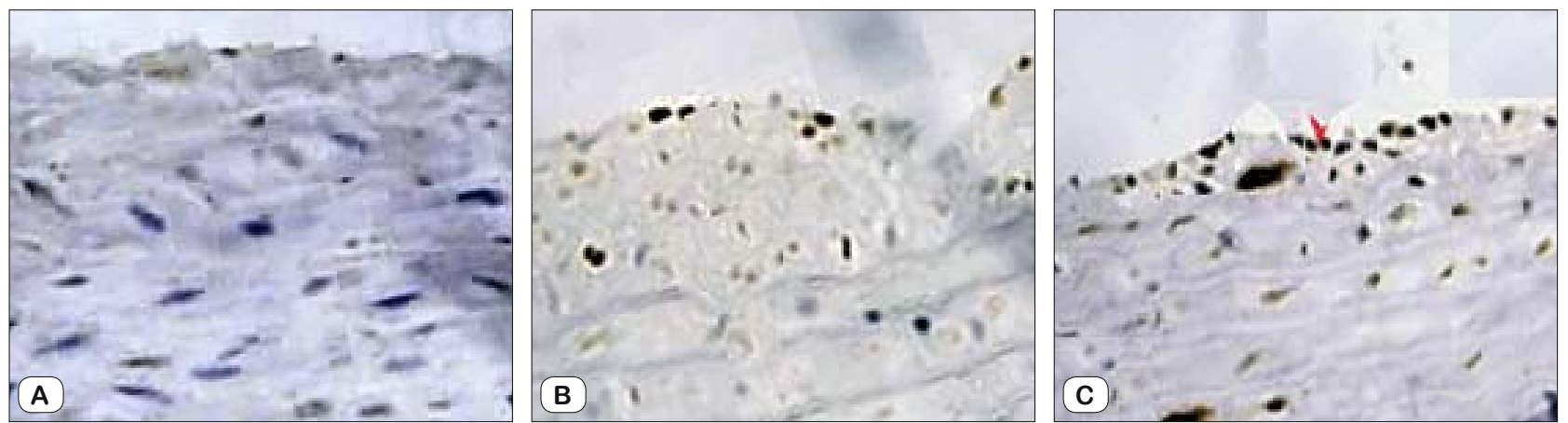

Fig. 3. P21 expressions in vessel wall after balloon injury. A - normal control group; B - postoperative 21st day status of the surgery group; $\mathrm{C}$ - postoperative 21 st day status of the piperonylic acid treatment group $(\mathrm{p}<0.01$ vs the surgery group).
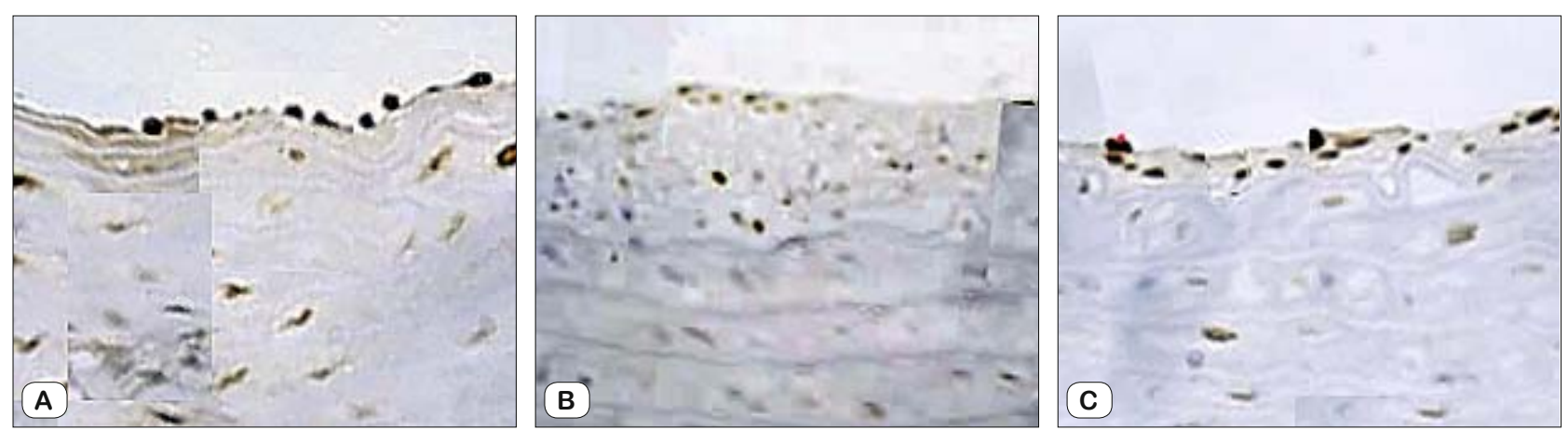

Fig. 4. P27 expressions in vessel wall after balloon injury. A - normal control group; B - postoperative 21st day status of the surgery group; $\mathrm{C}$ - postoperative 21 st day status of the piperonylic acid treatment group $(\mathrm{p}<0.01$ vs the surgery group)

\section{Discussion}

Stimulated by various factors, VSMCs have transformed from the non-proliferative contractile phenotype (high differentiation) to the proliferative type and they also have moved from the middle level to the intima. Excessive proliferation leads to the reconstruction of negative vessel and luminal stenosis. Besides, excessive proliferation of VSMCs contributes to the occurrence and development of many vascular proliferative diseases (9-11). Therefore, molecular biology studies regarding the proliferation and regulation of VSMCs have been spotlighted. Many factors stimulate the VSMCs proliferation through different pathways, which cooperate in the distribution pattern of network. The activated VSMCs complete division following the cell cycle, the blocking of which will effectively prevent and treat VSMCs proliferative diseases $(12,13)$.
Cyclin dependent kinase inhibitors (CKIs), which exist in the cell nucleus, dominate in negatively regulating the VSMCs proliferation cycle. CDKIs are highly expressed in static cells. However, the expression level plummets in case of division factors and injury of blood vessel, thus allowing PRB phosphorylation and normal cell cycle. On the other hand, P21 and P27 are the key members of CKI family. The overexpression of $\mathrm{P} 21$ inhibits the cell proliferation of mammals $(14,15)$. Mitrea et al. found that there was no P21 in normal or repaired artery, and P21 only existed in hyperplastic VSMCs. P21, which was transfected to VSMCs, was significantly expressed, which inhibited the proliferation of more than $90 \%$ of VSMCs. P27 mainly responds to the signals that accelerate or inhibit the proliferation outwards. It can specifically suppress the activation of cyclin/cyclin dependent kinase (CDK) complexes and block the progress of cell cycle (16). Some researches show 
that the expression levels of P27 vary depending on the phase of cell cycle. It is higher during the stationary phase and plummets in the midst of proliferation. Meanwhile, PCNA is the accessory protein of DNA polymerase $\delta$. It will promote the lengthening of DNA chain during DNA synthesis, so it is necessary for the cells to enter and survive the $\mathrm{S}$ phase. The expression of PCNA during the late G1 phase and S phase may be considered as a reliable indicator to reflect the degree of cell proliferation $(17,18)$.

Recently, people also have paid particular attention to the biological activity of piperonylic acid. Gomez-Garre et al confirmed that piperonylic acid regulated the blood lipid of an atherosclerosis animal model (19). Moreover, Gijsen et al worked on the mechanisms of piperonylic acid purification and antitumor activity (20). In addition, piperonylic acid can regulate lipid metabolism, cell cycle and apoptosis as well as protect vascular endothelial cells, suggesting that piperonylic acid can resist atherosclerosis. Nevertheless, relevant mechanisms remain limited $(21,22)$.

In summary, we found in this study that: 1) Piperonylic acid inhibited VSMCs excessive proliferation induced by FBS. Besides, the inhibitory effect was enhanced (20-80 mg/L) with increasing concentration. 2) In static VSMCs, P27 was highly expressed while P21 was lowly expressed. FBS suppressed the expression of P27 protein and obviously facilitated the VSMCs proliferation. 3) Piperonylic acid dramatically up-regulated the expression levels of P21 and P27 proteins, thus inhibiting the VSMCs proliferation and the neointimal hyperplasia after vascular injury, boosting the vascular reconstruction, and suppressing luminal stenosis eventually.

As a result, we have demonstrated that piperonylic acid resisted luminal stenosis of VSMCs excessive proliferation and we have also preliminarily explored the mechanism, verifying that piperonylic acid can reduce the PCNA expression and inhibit the G1/S phase of cell cycle by up-regulating the gene expressions of P21 and P27, thereby evidently inhibiting the VSMCs excessive proliferation. Given that endothelial cell injury, VSMCs proliferation signal transduction, and immunologic injury are the key mechanism and intervention targets for atherosclerosis, establishing atherosclerosis models, such as those of coronary atherosclerosis and balloon injury or restenosis after stent implantation for ApoE gene deficient mice and miniature pigs, will provide novel concepts and protocols for the prevention and treatment of atherosclerotic proliferative diseases by figuring out the optimum therapeutic dose of piperonylic acid and studying on the intervention targets on the levels of genes and molecules.

\section{References}

1. Liu J, Liu Y, Xu Y et al. Neurotransmitter noradrenaline downregulate cytoskeletal protein expression of VSMCs. Exp Mol Pathol 2012; 94: 79-83.

2. Shang YY, Si JQ, Li $\mathbf{L}$ et al. The effects of $\mathrm{pH} 0$ on electrophysiological properties of VSMCs in brain artery of spontaneously hypertensive rats. Zhongguo Ying Yong Sheng Li Xue Za Zhi 2012; 28: 268-270.

3. Shen X, Li H, Li W et al. Pioglitazone prevents hyperglycemia induced decrease of AdipoR1 and AdipoR2 in coronary arteries and coronary VSMCs. Mol Cell Endocrinol 2012; 363: 27-35.

4. Zheng B, Han M, Shu Y et al. HDAC2 phosphorylation-dependent Klf5 deacetylation and RARalpha acetylation induced by RAR agonist switch the transcription regulatory programs of p21 in VSMCs. Cell Res 2011; 21 : $1487-1508$.

5. Zhang HB, Wen JK, Zhang $\mathbf{J}$ et al. Flavonoids from Inula britannica reduces oxidative stress through inhibiting expression and phosphorylation of p47(phox) in VSMCs. Pharm Biol 2012; 49: 815-820.

6. Matos MA, Monte MJ, Sousa CC et al. Thermodynamic study of sesamol, piperonyl alcohol, piperonylic acid and homopiperonylic acid: a combined experimental and theoretical investigation. Org Biomol Chem 2010; 2: 908-914.

7. Begum N, Hockman S, Manganiello VC. Phosphodiesterase 3A(PDE3A) deletion suppresses proliferation of cultured murine vascular smooth muscle cells (VSMCs) via inhibition of mitogen-activated protein kinase (MAPK) signaling and alterations in critical cell cycle regulatory proteins. J Biol Chem 2011; 286: 26238-26249.

8. Liu Y, Wen JK, Dong LH et al. Kruppel-like factor (KLF) 5 mediates cyclin D1 expression and cell proliferation via interaction with c-Jun in Ang II-induced VSMCs. Acta Pharmacol Sin 2010; 31: 10-18.

9. Dong N, Zhu Q, Zhang P et al. Autophagy downregulates thrombin-induced VSMCs proliferation through lysosomal pathway. Int J Cardiol 2012; 159: $156-158$.

10. Luo X, Xiao Y, Song F et al. Increased plasma S-adenosyl-homocysteine levels induce the proliferation and migration of VSMCs through an oxidative stress-ERK1/2 pathway in apoE(-/-) mice. Cardiovasc Res 2012; 95: 241-250.

11. Smiljanic K, Dobutovic B, Obradovic M et al. Involvement of the ADAM 12 in thrombin-induced rat's VSMCs proliferation. Curr Med Chem 2012; 18: 3382-3386.

12. Liu A, Yang J, Huang $X$ et al. Relaxation of rat thoracic aorta by fibrate drugs correlates with their potency to disturb intracellular calcium of VSMCs. Vascul Pharmacol 2012; 56: 168-175.

13. Pawlinski R, Holinstat M. We can do it together: PAR1/PAR2 heterodimer signaling in VSMCs. Arterioscler Thromb Vasc Biol 2012; 31 : 2775-2776.

14. Yadav V, Sultana S, Yadav J et al. Gatifloxacin Induces $S$ and G(2)Phase Cell Cycle Arrest in Pancreatic Cancer Cells via p21/p27/p53.PLoS One 2012; 7: 796-799.

15. Szentmary N, Stundl A, Szende B et al. P21, p27, bax, cathepsin and survivin pathways in macular dystrophy corneas. Histol Histopathol 2010; 25: $287-290$.

16. Mitrea DM, Yoon MK, Ou L et al. Disorder-function relationships for the cell cycle regulatory proteins p21 and p27. Biol Chem 2012; 393: 259-274.

17. Yoon MK, Mitrea DM, Ou L et al. Cell cycle regulation by the intrinsically disordered proteins p21 and p27. Biochem Soc Trans 2012; 40: 981-988.

18. Chen J, Amos CI, Merriman KW et al. Genetic variants of $\mathrm{p} 21$ and p27 and pancreatic cancer risk in non-Hispanic Whites: a case-control study. Pancreas 2010; 39: 1-4.

19. Gomez-Garre D, Estrada V, Ortega-Hernández A et al. Authors' Reply: Association of HIV-Infection and Antiretroviral Therapy with Levels of Endothelial Progenitor Cells and Subclinical Atherosclerosis. J Acquir Immune Defic Syndr 2012; 62: 23-25.

20. Gijsen F, van der Giessen $A$, van der Steen $A$ et al. Shear stress and advanced atherosclerosis in human coronary arteries. J Biomech 2012; 11 : 58-62.

21. Humphries SE. Out with print in with e publication: Editor in Chief's Report for Atherosclerosis for 2012. Atherosclerosis 2012; 226: 1-2.

22. Winnik S, Stein S, Matter CM. SIRT1 - An Anti-Inflammatory Pathway at the Crossroads Between Metabolic Disease and Atherosclerosis. Curr Vasc Pharmacol 2012; 10: 693-696.

Received May 13, 2013. Accepted July 25, 2014. 